\section{Reducing mortality in newly diagnosed standard-risk acute promyelocytic leukemia in elderly patients treated with arsenic trioxide requires major reduction of chemotherapy: a report by the French Belgian Swiss APL group (APL 2006 trial)}

Acute promyelocytic leukemia (APL) is a specific subtype of acute myeloid leukemia (AML) characterized by its morphology, presence of $t(15 ; 17)$ and high sensitivity to the differentiating effect of ATRA (all-trans retinoic acid) and the pro-apoptotic effect of ATO (arsenic trioxide). ${ }^{1}$ The combination of ATRA and anthracycline-based chemotherapy (CT) has been the mainstay of newly diagnosed APL treatment over the last two decades. ${ }^{1-3}$ It yields complete remission (CR) rates of about $90 \%$, and less than $10 \%$ of standard-risk APL (i.e., with baseline white blood count (WBC) below $10 \mathrm{G} / \mathrm{l})$ relapse, a relapse rate that may remain somewhat higher in high-risk APL (with baseline WBC greater than $10 \mathrm{G} / \mathrm{l}$ ).

Contrary to other types of AML, APL is not frequent in the elderly. Only $15-20 \%$ and $1-6 \%$ of APL patients are older than 60 and 70 years in published APL series, respectively, although this could in part reflect poor inclusion of elderly APL patients in clinical trials. ${ }^{4,5}$ Treatment of APL in the elderly with conventional ATRA-CT regimens is associated, like in younger patients, with very few relapses, but relatively high death rates during induction treatment or in CR. ${ }^{6}$ Indeed, in our published APL 2000 trial, the CR rate in patients older than 60 was $98.1 \%$, and the cumulative incidence of relapse (CIR), event-free survival (EFS) and overall survival (OS) rates were $9.4 \%, 79.1 \%$, and $88.3 \%$, respectively. ${ }^{7}$ This compared with $99 \% \mathrm{CR}, 4.7 \% \mathrm{CIR}$ and $97.9 \%$ OS in patients younger than 65 included in the same clinical trial. ${ }^{7}$ Of note, among older patients, $5 / 51(10 \%)$ died in CR.

Recent results have shown that, in standard risk APL, ATRA-ATO combinations (without CT) are at least as effective as classical ATRA-CT regimens while being less myelosuppressive ${ }^{8-10}$ thus constituting a very appealing approach to treat elderly patients. However, when our APL 2006 trial started, the feasibility of APL treatment without CT was unknown. Furthermore, access to ATO still remains limited for frontline treatment of APL in most countries. Finally, while intuitively ATRA-ATO regimens would improve the outcome in elderly APL compared with ATRA-CT, no large series of elderly patients who received ATO during first-line treatment have yet been published. ${ }^{10,11}$ We present here the results of our APL 2006 trial where we combined ATO to ATRA and reduced CT in standard-risk APL patients older than 70 .

Between 2006 and 2015, newly diagnosed patients with documented APL (subsequently confirmed by cytogenetics and/or molecular biology) aged $>70$ years and presenting with $\mathrm{WBC}<10 \mathrm{G} / \mathrm{L}$ were eligible for APL 2006 trial. They were included in the elderly standard-risk group after informed consent was obtained. They received induction treatment with ATRA $45 \mathrm{mg} / \mathrm{m}^{2} / \mathrm{d}$ until CR and Idarubicin (Ida) $9 \mathrm{mg} / \mathrm{m}^{2} / \mathrm{d}$ on days 3,5 and 7; a first consolidation course with Ida $9 \mathrm{mg} / \mathrm{m}^{2} / \mathrm{d}$ for 3 days combined with ATO $0.1 \mathrm{mg} / \mathrm{kg} / \mathrm{d}$ for 25 days; a second consolidation course with ATO (same schedule) and ATRA for 15 days. Patients then received maintenance treatment for 2 years with intermittent ATRA, continuous 6-MP (6-mercaptopurine) and MTX (methotrexate), plus 15 day ATO cycles every 3 months for the first year. In September 2010, after inclusion of 55 patients, because mortality in CR was still high (10 of the 51 patients who had achieved CR) while no relapse was
Table 1. Baseline characteristics of standard risk APL patients older than 70 years.

\begin{tabular}{lc} 
Median [Q1-03] & $N^{=}=123$ \\
Age $(\mathrm{y})$ & $73.5[71.8 ; 77.9]$ \\
WBC $(\mathrm{G} / \mathrm{L})$ & $1.1[0.8 ; 1.8]$ \\
\hline Platelets $(\mathrm{G} / \mathrm{L})$ & $44.0[22.5 ; 87.5]$ \\
Fibrinogen $(\mathrm{g} / \mathrm{l})$ & $2.3[1.6 ; 3.3]$ \\
\%M3v & $5 \%$ \\
\%Previous cancer & $35 \%$ \\
\hline WBC: white blood cells &
\end{tabular}

observed, consolidation CT was reduced to one day of Ida during the first consolidation cycle. The primary endpoint was EFS from CR achievement. Relapse, survival, side effects of the treatment and duration of hospitalization were secondary endpoints.

Analysis was performed on a modified intent-to-treat principle, excluding only diagnostic errors and withdrawals of consent. Censored endpoints were estimated by the nonparametric Kaplan-Meier method ${ }^{12}$ and then compared between groups by the log-rank test, after checking for proportional hazards. In estimating relapses, we took into account, for competing risks, deaths in first CR using the cumulative incidence curves and then compared results using the Gray test,,$^{13}$ whereas the Fine and Gray model was used to estimate subdistribution HR. We present here the results at the reference date of January 1 , 2017.

Median age of the 123 included patients was 73.5 years (range $70-88.4$ ) with $56 \%$ males (Table 1). Of note, $35 \%$ of the patients had a previous history of treated cancer $41 \%$ of them had a previous history of prostate cancer which was the most prevalent tumor followed by $20 \%$ with history of breast carcinoma, and were thus potentially therapy-related APL. Of the patients, $43.5 \%$ were classified as low risk disease according to Sanz score. A total of $113(92 \%)$ patients achieved CR, $4(3 \%)$ had resistant leukemia and $6(5 \%)$ had early death from sepsis $(n=3)$ or multiorgan failure $(n=3)$. Of the 113 patients, 45 received the 3 -day Ida consolidation cycle planned at trial onset, and the subsequent 68 patients were planned to receive the attenuated 1-day cycle. By the end of the second consolidation course, all patients achieved negative PML/RARA minimal residual disease. Only 3 relapses were seen among the 113 patients who achieved CR (5year CIR of $2.9 \%$ ), including 0 with the original and 3 with the amended protocol ( $P=0.10$, Gray's test). Fourteen (12\%) patients died in CR: among them, $4(4 \%)$ were included after versus $10(20 \%)$ included before the amendment ( $P=0.045$, Fisher's test). Causes of death in CR were sepsis ( $n=4$ before and 2 after amendment), bleeding ( $n=5$ before and 1 after), general deterioration ( $\mathrm{n}=1$ before), and prostate cancer ( $\mathrm{n}=1$ after).

The 5 -year OS was $80 \%$ (95\%CI, 73-88\%) in the overall population and $75.5 \%$ and $84.7 \%$, respectively, before and after the amendment ( $P=0.55$ by the log-rank test). Similarly, 5-year EFS was $80 \%$ (95\%CI, 73-88\%) in the overall population and $79.5 \%$ and $81.5 \%$, respectively, before and after the amendment $(P=0.80$ by the log-rank test, respectively) (Figure 1). Median time to absolute neutrophil count $>1 \mathrm{G} / \mathrm{L}$ and platelets $>50 \mathrm{G} / 1$ after the first consolidation course was 16.2 and 11.9 days in the original regimen versus 5.6 and 4.0 days in the amended protocol $(P<0.0001$ and $P<0.0001$, respectively), while the second consolidation course, with ATO alone, was not associated with myelosuppression. 


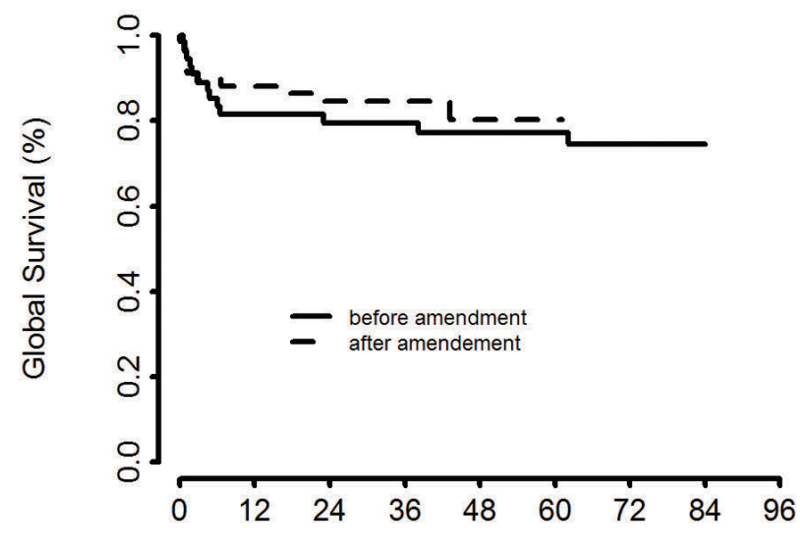

Time (months)

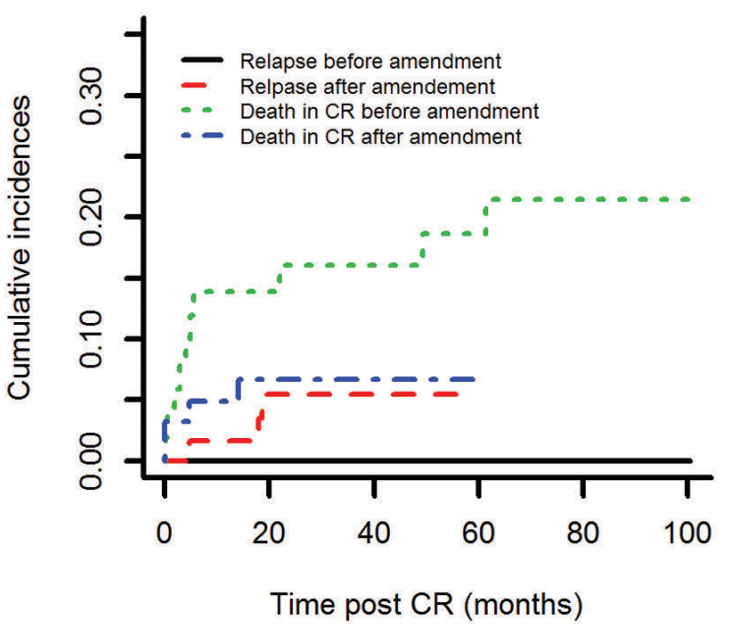

We therefore observed that, as in younger patients, introduction of ATO in the frontline treatment of APL in elderly allowed reduction of the CT regimen without an increase in the relapse rate. However, in order to obtain a significant reduction in mortality in CR, the amount of CT had to be drastically reduced to only one day of Idarubicin during the consolidation cycle. Indeed, using a single 3-day Idarubicin consolidation cycle did not significantly decrease the rate of deaths in CR as compared with our previous APL 2000 trial where elderly patients received 2 cycles of Daunorubicin $\left(60 \mathrm{mg} / \mathrm{m}^{2} / \mathrm{d}\right.$ x3 \& 45 $\mathrm{mg} / \mathrm{m}^{2} / \mathrm{d} \mathrm{x} 3$ ) without AraC for consolidation. A possible difference, however, with the APL 2000 trial is that the APL 2006 trial was modified above the age of 70 while the precedent was modified above the age of 60 .

Our results contrast with those of Sanz et al. ${ }^{3}$ who, for consolidation cycles in elderly, moderately reduced anthracycline dose in comparison with younger patients: Ida $5 \mathrm{mg} / \mathrm{m}^{2} / \mathrm{d} \mathrm{x} 4$ days for the first consolidation cycle (instead of $7 \mathrm{mg} / \mathrm{m}^{2} / \mathrm{d} \mathrm{x} 4$ ), mitoxantrone for 3 days for the second cycle (unchanged) and Ida $12 \mathrm{mg} / \mathrm{m}^{2} / \mathrm{d}$ x1 day (instead of 2 days) for the last cycle. This modest reduction allowed them to reduce mortality in elderly patients from 18 to $5 \%$. $^{14}$

Recent results obtained in adult APL with ATRA-ATO regimens (CT free) in the MRC and in the Italian-German

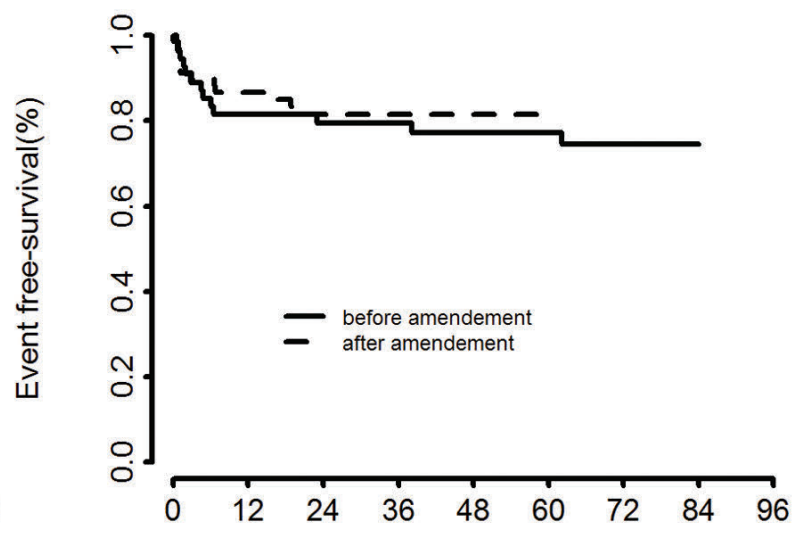

Time (months)
Figure 1. Survival curves of elderly treated in the APL 2006 trial. A and B represent overall survival and event-free survival in elderly, respectively, before and after the amendment. $\mathrm{C}$ represents cumulative incidence of relapse and death in CR of elderly patients before and after the amendment.
APL0406 trials suggest that those regimens may further decrease the incidence of deaths in CR, without increasing relapses. However, only 49 patients older than 60 years had been enrolled in the MRC trial - where the long-term survival rate of elderly was $75 \%,{ }^{10}$ and no patint older than 70 was included in APL0406. In addition, the potential of ATRA-ATO regimens to increase $\mathrm{CR}$ rates must be validated in geriatric series. Actually, CR rate was high $(92 \%)$ in our series with ATRA and 3 days of Idarubicin. Causes of failure were early death associated with myelosuppression ( $5 \%$ of the patients) and leukemic resistance (3\%), two causes that would potentially be reduced by CT-free regimens. Conversely, ATRA-ATO regimens might also be associated with potentially fatal complications in the elderly, especially early severe activation syndrome and later cardiac or neurological impairment, due to high cumulative doses of ATO.

Ramy Rahmé, Lionel Ades, ${ }^{1}$ Xavier Thomas, ${ }^{2}$ Agnès Guerci-Bresler, ${ }^{3}$ Arnaud Pigneux, ${ }^{4}$ Norbert Vey, Emmanuel Raffoux, ${ }^{1}$ Sylvie Castaigne, ${ }^{6}$ Olivier Spertini, ${ }^{7}$ Sebastian Wittnebel, Jean Pierre Marolleau," Gandhi Damaj, ${ }^{10}$ Dominique Bordessoule, 11 Julie Lejeune, ${ }^{12}$ Sylvie Chevret ${ }^{12}$ and Pierre Fenaux ${ }^{1}$

${ }^{1}$ Hôpital Saint Louis, Université Paris Diderot, France; ${ }^{2} H o s p i c e s$ Civils de Lyon, Département d'Hématologie, Centre Hospitalier 
Lyon-Sud, Pierre Bénite, France; ${ }^{3}$ Hematology Department, Nancy University Hospital, France; ${ }^{4}$ Hematology Department, Bordeaux University Hospital, France; 'Institut Paoli Calmette, Marseille, France; ${ }^{6}$ Hematology Department, Versailles University Hospital, France; 'Hematology Department, University of Lausanne, CHUV, Switzerland; ${ }^{8}$ Deparment of Clinical and Experimental Hematology, Institut Jules Bordet (ULB), Brussels, Belgium; ${ }^{9}$ Hematology Department, Amiens University Hospital, France; ${ }^{10}$ Hematology Department, Caen University Hospital, France; "Hematology Department, Limoges University Hospital, France and ${ }^{12}$ Service de Biostatistique et Information médicale, Hôpital Saint Louis, Université Paris Diderot, France

Funding: this study was supported by the programme hospitalier de recherche clinique and the Association pour la Recherche sur le Cancer (ARC).

Correspondence:pierre.fenaux@aphp.fr doi:10.3324/haematol.2018.193151

Information on authorship, contributions, and financial \& other disclosures was provided by the authors and is available with the online version of this article at www. haematologica.org.

\section{References}

1. Sanz MA, Grimwade D, Tallman MS, et al. Management of acute promyelocytic leukemia: recommendations from an expert panel on behalf of the European LeukemiaNet. Blood. 2009;113(9):1875-1891.

2. Adès L, Chevret S, Raffoux E, et al. Long-term follow-up of European APL 2000 trial, evaluating the role of cytarabine combined with ATRA and Daunorubicin in the treatment of nonelderly APL patients. Am J Hematol. 2013;88(7):556-559.

3. Sanz MA, Montesinos P, Rayón C, et al. Risk-adapted treatment of acute promyelocytic leukemia based on all-trans retinoic acid and anthracycline with addition of cytarabine in consolidation therapy for high-risk patients: further improvements in treatment outcome. Blood. 2010;115(25):5137-5146.

4. Lehmann S, Ravn A, Carlsson L, et al. Continuing high early death rate in acute promyelocytic leukemia: a population-based report from the Swedish Adult Acute Leukemia Registry. Leukemia. 2011;25(7):1128-1134.

5. Rahmé R, Thomas X, Recher C, et al. Early death in acute promyelocytic leukemia (APL) in French centers: a multicenter study in 399 patients. Leukemia. 2014;28(12):2422-2424.

6. Ades L, Chevret S, De Botton S, et al. Outcome of acute promyelocytic leukemia treated with all trans retinoic acid and chemotherapy in elderly patients: the European group experience. Leukemia. 2005; 19(2):230-233.

7. Adès $\mathrm{L}$, Chevret $\mathrm{S}$, Raffoux $\mathrm{E}$, et al. Is cytarabine useful in the treatment of acute promyelocytic leukemia? Results of a randomized trial from the European Acute Promyelocytic Leukemia Group. J Clin Oncol. 2006;24(36):5703-5710.

8. Lo-Coco F, Avvisati G, Vignetti M, et al. Retinoic acid and arsenic trioxide for acute promyelocytic leukemia. N Engl J Med. 2013; 369(2):111-121.

9. Platzbecker U, Avvisati G, Cicconi L, et al. Improved outcomes with retinoic acid and arsenic trioxide compared with retinoic acid and chemotherapy in non-high-risk acute promyelocytic leukemia: final results of the randomized Italian-German APL0406 trial. J Clin Oncol. 2017;35(6):605-612.

10. Burnett AK, Russell NH, Hills RK, et al. Arsenic trioxide and all-trans retinoic acid treatment for acute promyelocytic leukaemia in all risk groups (AML17): results of a randomised, controlled, phase 3 trial. Lancet Oncol. 2015;16(13):1295-1305.

11. Kota V, Karkhanis P, Sharma R, et al. A prospective multi-center trial shows reduction of early deaths (ED) and improved survival in elderly acute promyelocytic leukemia (APL) patients ( $>60$ years). Results of using a simplified treatment algorithm and expert support in georgia, South Carolina and neighboring states. Blood. 2016;128(22):1622.

12. Kaplan EL, Meier P. Nonparametric estimation from incomplete observations. J Am Stat Assos. 1958;53457-481.

13. Fine J, Gray R. A proportional hazards model for the subdistribution of a competing risk. J Am Stat Assoc. 1999;94(446):496-509.

14. Martínez-Cuadrón D, Montesinos P, Vellenga E, et al. Long-term outcome of older patients with newly diagnosed de novo acute promyelocytic leukemia treated with ATRA plus anthracycline-based therapy. Leukemia. 2018;32(1):21-29. 